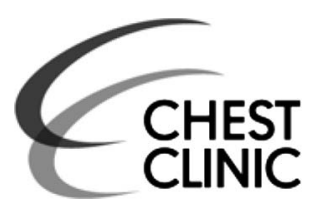

${ }^{1}$ Aintree Health Outcomes Partnership, University of Liverpool, Liverpool, UK ${ }^{2}$ Aintree Chest Centre, University Hospital Aintree, Liverpool, UK

${ }^{3}$ Respiratory Programme, Department of Health, London, UK

\section{Correspondence to}

Dr Paul Phillip Walker, Aintree Chest Centre, University Hospital Aintree, Lower Lane, Liverpool L9 7AL, UK; ppwalker@liv.ac.uk

Received 1 July 2012 Revised 1 May 2013 Accepted 25 May 2013 Published Online First 20 June 2013

\section{SLinked}

- http://dx.doi.org/10.1136/ thoraxjnl-2013-203717

To cite: Walker $\mathrm{PP}$, Thompson $\mathrm{E}$, Crone $\mathrm{H}$, et al. Thorax 2013;68:968-970.

\title{
Use of mortality within 30 days of a COPD hospitalisation as a measure of COPD care in UK hospitals
}

\author{
P P Walker, ${ }^{1,2}$ E Thompson, ${ }^{1}$ H Crone, ${ }^{1} \mathrm{G} \mathrm{Flatt}_{1}{ }^{1} \mathrm{~K}$ Holton, ${ }^{3} \mathrm{~S}$ L Hill, ${ }^{3}$ M G Pearson ${ }^{1,2}$
}

\begin{abstract}
Mortality rate has been proposed as a metric of hospital chronic obstructive pulmonary disease (COPD) care in light of variation seen in national COPD audits. Using Hospital Episode Statistics (hospital 'coding') we examined 30-day mortality after COPD hospitalisation in 150 UK hospitals during 2006-2007 and 2007-2008. Mean and median 30-day mortalities were similar each year but the coefficient of variation was $>20 \%$ and hospitals could change from a low or high quartile to the median by chance. We could not detect any reasons for hospitals being at the extremes. 30-day mortality after COPD hospitalisation is a complex variable and unlikely to be useful as a primary annual COPD metric.
\end{abstract}

\section{INTRODUCTION}

Guidelines describe best care for people with COPD and the UK has a national programme to drive care quality. In stroke care and cardiac thrombolysis national audits have developed and used metrics, as part of national strategies, for improving outcomes. ${ }^{1}$ There are similar examples from outside the UK. However, finding metrics with which to measure COPD care has proved difficult.

The 2003 and 2008 National COPD Audits (NCROP) reported wide variation in hospital and 90-day mortality rates and the 2003 audit showed lower mortality in hospitals with more consultants and a better 'organisational score'. ${ }^{2}$ Mortality is undoubtedly an important metric; however, its utility requires separation of deaths that are potentially 'preventable' from the majority that are 'inevitable' due to more severe disease and this has previously not proved possible even with the detailed NCROP dataset. ${ }^{3}$

The English Hospital Episodes Statistics (HES) database includes large numbers of COPD cases and allows examination of variation in mortality rate between hospitals and factors which contribute to this. HES data is already being used to assess quality of care in other diseases and related to certain procedures. In order to determine whether mortality is a potentially useful annual metric of hospital COPD care we examined COPD deaths during and after hospitalisation in 2006-2007 and 2007-2008 to quantify how much between hospital variation in 30-day mortality remains after correction for age, comorbidity and deprivation, the year-on-year variation and consequently whether all cause 30-day mortality represents a potentially useful annual metric of hospital COPD care quality.

\section{METHODS}

All National Health Service (NHS) hospitals in England submit information about each admission. These anonymised hospital episode statistics contain a limited clinical dataset including up to 14 International Classification of Diseases-10 diagnosis codes and the linked alive/dead status (and date of death) from the Office for National Statistics. We included all adult (16 years + ) emergency admissions to medical specialties in 150 acute hospitals over 2 years (2006/2007 and 2007/2008).

\section{Data extraction}

A clinical algorithm defined when COPD was likely to be the primary reason for admission based on the first three codes in the HES diagnoses list. This included all admissions where a COPD code was first coded at position $1(\mathrm{P} 1=$ coded at position 1$)$ plus admissions with COPD coded at P2 or P3 and either pulmonary infection code(s), pulmonary symptom code(s) or a combination at P1-2. If another major diagnostic code, such as myocardial infarction, was recorded before a COPD code this was not classified a COPD admission. If coded at P4 or lower COPD was almost never the primary reason for admission.

\section{Data corrections}

Age, gender and the Index of Multiple Deprivation are standard fields in HES. Index of multiple deprivation (IMD) is determined from patient postcode. The Charlson comorbidity score ${ }^{4}$ modified by excluding COPD was determined from the other International Classification of Diseases-10 codes. Previous admission rate was the number of COPD hospitalisations in the 12 months prior to the first admission in 2007-2008. Age and gender datasets were $>99.98 \%$ complete but postcode and therefore IMD was missing in 1\% (2536).

We determined the effect of age, deprivation, comorbidity, gender and previous admission rate on all cause 30-day mortality and used age, deprivation and comorbidity to correct each hospital's data against the national picture. As the univariate relationships were not linear we calculated mean 'expected' 30-day mortality from the full dataset at patient level, for example for 5-year age categories (55-59 years, 60-64 years, etc), then applied this to all admissions to calculate 'expected' mortality for each hospital. This was then compared with the 'observed' mortality to determine a correction for age-adjustment at hospital level which was applied 
to measured 30-day mortality. We followed the same process for deprivation by ranking scores for each patient, dividing the dataset into quintiles and applying the correction factor for each quintile. We used the Charlson index ${ }^{4}$ to correct for comorbidity by grouping the scores into no (Charlson 0 ), some (1-2) or significant comorbidity $(3+)$ and applying the correction. Where diabetes, liver disease and solid organ tumour were recorded severity could not be derived so these were scored according to presence or absence.

\section{Statistical analysis}

Data were analysed using SPSS V.17.0. Analyses were performed on the whole 2-year cohort unless stated. Pearson's correlation coefficient was used to examine the association between parameters. Factors associated with 30-day mortality were identified by univariate analysis and multiple logistic regression models. A funnel plot was created to illustrate between hospital mortality variations.

\section{RESULTS}

\section{Factors affecting 30-day mortality}

There were 125113 emergency COPD admissions in 20062007 and 123883 in 2007-2008. There were 27796 deaths during 2006-2008 with a mean 93 (SD 52) annual deaths per hospital and a mean 830 (462) COPD admissions.

Higher age, male gender, lesser deprivation and more comorbidity were associated with higher 30-day mortality. The strongest factor was age $-21 \%$ mortality aged $85+$ years versus $2.6 \%<55$ years (mortality $\mathrm{OR}=8.54$ (CI 7.72 to 9.44), $\mathrm{p}<0.001$ ). Deprivation (mortality $\mathrm{OR}=1.15$ (CI 1.11 to 1.2 ), $\mathrm{p}<0.001$ least deprived vs most deprived quintile), comorbidity (mortality $\mathrm{OR}=2.1$ (CI 2.03 to 2.17 ), $\mathrm{p}<0.001$ Charlson score $3+$ vs 0 ) and gender (mortality $\mathrm{OR}=1.05$ (CI 1.02 to 1.08), $\mathrm{p}<0.001$ male vs female) were all highly significant. Dividing hospitals into quintiles by the mean IMD of their COPD admissions showed that in hospitals with the most deprived patients COPD formed $6.3 \%$ of all emergency admissions with a lower mean age of 71.2 years, more admissions per patient (1.72) and a lower mortality of $9.0 \%$ compared with hospitals with the least deprived patients where the figures were 4.0\%, 73.8 years, 1.44 and $12.9 \%$, respectively (all univariate trends $\mathrm{p}<0.001$ ).

\section{Variation between hospitals}

Median 30-day mortality was 11.3\% (IQR 9.9-12.6) in 20062007 and 11.4\% (IQR 9.7-12.9) in 2007-2008. Despite adjustment for age, deprivation and comorbidity considerable residual variation remained with $27 \%$ (41/150) hospitals having mortality outside (17 above and 24 below) the 3 SD lines of a funnel plot (figure 1$).^{5}$

\section{Between-year variation}

The mean difference in 30-day mortality between years is small $(0.1 \%)$ and there is a modest correlation $(r=0.52, p<0.01)$ for hospital rates in 1 year to be similar in the next. However the coefficient of variation in hospital mortality between years is $20.1 \%$. Sixteen trusts had year-on-year mortality rates that exceeded 1.67 SDs (3.8\% year-on-year variation) with nine increasing and seven decreasing such that it is possible to be an extreme outlier in 1 year yet close to the median the next. In part this reflects the small number of deaths such that for a majority of hospitals a $3.8 \%$ variation represents only $8-30$ deaths.

High mortality appeared independent of hospital size, number and burden of COPD admissions, respiratory specialist input (specialty of the discharge physician) and length of stay.

\section{DISCUSSION}

Death during or within 30 days of a COPD admission is common and because it is measurable it is a potential quality indicator. There is wide residual variation between hospitals even after control for age, deprivation and comorbidity such that a quarter of hospitals are outliers on a 2-year standard funnel plot implying factors other than chance at work. However, while care quality might be a factor there are many other potential explanations that are either unmeasured (case severity) or uncontrolled for (admission thresholds) which limits its value as an indicator.

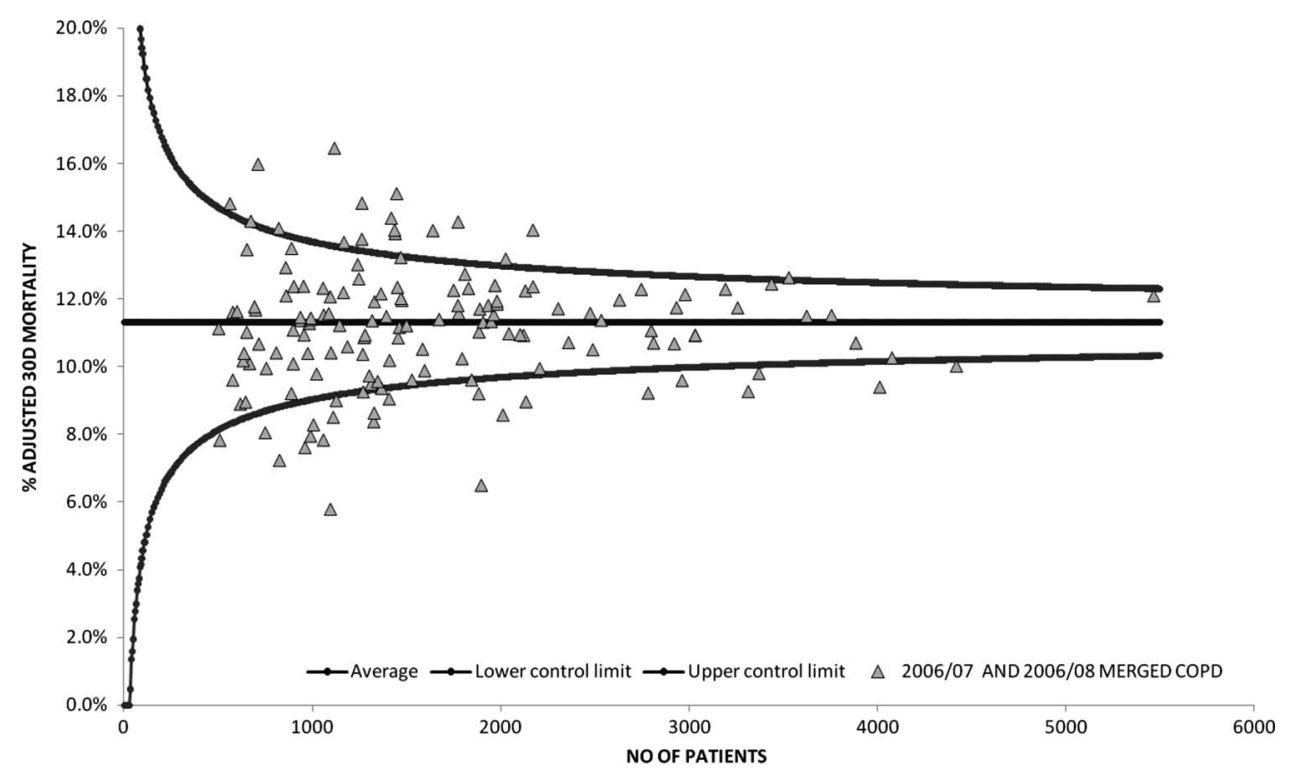

Figure 1 A funnel plot of mean 30-day mortality plotted against mean annual number of COPD admissions for all hospitals in England during 2006-2008. 
The 2003 NCROP audit suggested a lower death rate in larger units with more staff and facilities implying potentially avoidable deaths. Between 2006-2007 and 2007-2008 there had been no major initiatives expected to alter care and no change in HES coding so we might have expected some consistency in mortality rates. However, while national mean values for years 1 and 2 are similar and individual hospital values correlate statistically there is high variance of between-hospital values with as many rising as falling. For a given hospital to be considered statistically different from 1 year to the next a $3.8 \%$ change in mortality rate $(1.67 \mathrm{SD})$ or a more than $40 \%$ shift from the mean is necessary. That alone makes year-on-year data challenging to interpret.

While many COPD deaths are inevitable the proportion that is preventable remains unknown and may vary with non-medical care factors. Thus, good practice such as admission prevention schemes could perversely increase hospital per cent mortality by not admitting those more likely to survive; although absolute death numbers for the population would remain similar. Conversely a hospital that succeeded in keeping patients with the most severe disease alive would look 'good' in that year, but the natural history cannot be radically changed and those ill patients will be added to the next few years' mortality data. Assessment of mortality over a 3-5 years period would have less variability and might identify a few hospitals with persistently high mortality but could still not correct for the balance between community and hospital while defeating the intent to have an annual metric. Additionally it is vital that attempts to reduce mortality do not contribute towards failure to provide adequate end-of-life care to patients where death is inevitable.

Strengths of the HES database are inclusion of all emergency admissions and our case selection algorithm was designed to include all inpatients that clinicians would recognise as having COPD. Thus COPD coded at positions 2 or 3 with a respiratory infection (bronchitis or pneumonia) at position 1 would have been entered by respiratory clinicians into the 2008 NCROP audit as a COPD exacerbation. Omission of cases where COPD was second or third excludes 19\% of COPD admissions and $40 \%$ of deaths resulting in a mortality rate considerably lower than NCROP or death certification. While our coding definitions are new they have been presented, discussed and agreed with UK COPD specialists and match national audit data. Using linked 30-day mortality creates an outcome not dependent on length of stay, collected robustly by the Office for National Statistics, comparable for all hospitals and includes all-cause deaths including cardiovascular disease. However our analyses have been repeated using inhospital and 90-day mortality with similar results.

Limitations of HES are that we have no specific measures of COPD severity (spirometry or blood gases) and diagnosis depends on coding; an area we have tried to improve upon by using three rather than only the first diagnosis code. The univariate analysis (confirmed in logistic regression) showing the most deprived patients had lesser mortality was a surprise but there are pointers that the most deprived patients are younger and have more admissions per year suggesting that the case mix within the denominator may vary with deprivation. Deprivation is not a significant predictor if the logistic regression is repeated using only last admission for each patient but UK hospital indicators are based on analysis by admissions rather than by individuals.

In conclusion 30-day mortality after hospitalisation is too variable to be a reliable annual metric of hospital COPD care as there appears to be considerable year-on-year variation related to case mix rather than care delivery. Due to an absence of disease severity markers it is impossible to distinguish avoidable from inevitable deaths and the analyses provide no indication of what aspect of healthcare should be changed. Moreover even when a death is avoided disease progression continues unchanged so, unlike asthma or a revascularised heart, the benefits are not long lived. More worryingly if it were to be adopted we would need to understand the reasons for variability and what a hospital should do to improve or much effort might have no detectable benefit. It might also mean that obvious good practice, such as admission prevention schemes, could be unsupported. This is not to argue that metrics should not be applied rather that annual mortality is a poor COPD metric; something which would also be true for certain other chronic diseases. Use of specific therapy such as non-invasive ventilation might represent a useful hospital metric but it is not reliably coded (only 30\% coding in our institution). A metric we have suggested previously is bed days per unit population controlled for prevalence. This reflects patients' desire to be managed at home, the state's desire to reduce hospitalisation and requires a joint community hospital approach; however, there may be others.

Acknowledgements We would like to thank Dr K Bodger for his advice and assistance in revising the manuscript.

Contributors Extraction and manipulation of data, analysis and statistical analysis - PPW, ET, HC, GF and MGP. Interpretation of data and further analysisPPW, KH, SLH and MGP. Writing, editing and revising manuscript—PPW, KH, SLH and MGP.

Funding The funding for this work was provided by the Department of Health, London, UK.

\section{Competing interests None.}

Provenance and peer review Not commissioned; externally peer reviewed.

Data sharing statement Complete data and methodology from this work has been shared with the Department of Health and the National COPD Strategy Leads/team.

\section{REFERENCES}

1 Quality and Performance in the NHS: High Level Performance Indicators and Clinical Indicators. Department of Health, 2001. http://www.performance.doh.gov.uk/indicat/ nhsci.pdf (accessed Sept 2011)

2 Price LC, Lowe D, Hosker HS, et al.; for the British Thoracic Society and the Royal College of Physicians Clinical Effectiveness Evaluation Unit (CEEu). UK National COPD Audit 2003: Impact of hospital resources and organisation of care on patient outcome following admission for acute COPD exacerbation. Thorax 2006;61:837-42.

3 Lilford R, Pronovost P. Using hospital mortality rates to judge hospital performance: a bad idea that just won't go away. BMJ 2010;340:c2016.

4 Charlson ME, Pompei P, Ales KL, et al. A new method of classifying prognostic co-morbidity in longitudinal studies: development and validation. J Chronic Dis 1987:40:373-83.

5 Jones HE, Ohlssen DI, Spiegelhalter DJ. Use of the false discovery rate when comparing multiple health care providers. J Clin Epidemiol 2008;61:232-40. 